\title{
Greenness, Perceived Pollution Hazards and Subjective Wellbeing: Evidence from China
}

Wenjie $\mathrm{WU}^{1}$, Yanwen $\mathrm{YUN}^{1}$, Bo $\mathrm{HU}^{2}$, Yeran $\mathrm{SUN}^{3}$, Yang Xiao ${ }^{4 *}$

${ }^{1}$ College of Economics, Jinan University, No. 601 Huangpu Road West, Guangzhou, 510632, China. Email: wenjiewu@jnu.edu.cn; yanweny520@yeah.net

${ }^{2}$ Care Policy and Evaluation Centre, Department of Health Policy, London School of Economics and Political Science, London WC2A 2AE, United Kingdom. Email: b.hu@1se.ac.uk

${ }^{3}$ Department of Geography, College of Science, Swansea University, Swansea SA28PP, United Kingdom. Email: yeran.sun@swansea.ac.uk

* Corresponding author:

Yang XIAO, Department of Urban Planning, College of Architecture and Urban Planning, Tongji University, Shanghai, China, email: yxiao@tongji.edu.cn

This paper has not been published previously, and it is not under consideration for publication elsewhere. Its publication is approved by all authors. 


\title{
Greenness, Perceived Pollution Hazards and Subjective Wellbeing: Evidence from China
}

\begin{abstract}
Urbanisation from the developing world has been phenomenal and renewed the interest of studying the connection between urban greenness and subjective wellbeing. This paper responds to this greenness-wellbeing connection by shifting its focus towards systematically exploring the influences of urban greenness, perceived pollution hazards, and their interaction terms on subjective wellbeing. Using a combination of green view data and individual survey data in Beijing, we find that perceived pollution hazards about the disposal of waste, polluted water, and air pollution have significant interaction effects with eye-sensored greenness exposures on subjective wellbeing. Findings of this study suggest that policies geared towards mitigating particular domains of pollution hazards and improving green landscape should work together for shaping people's quality of life.
\end{abstract}

Keywords: residential greenness, subjective wellbeing, pollution hazards 


\section{Introduction}

A substantial share of urban greenness exposures takes place in the outdoor context, where people work and live predominantly in cities for the opportunities of social interactions and leisure. In this context, greenness exposures have been an underlying channel that affect the degree of residents' subjective wellbeing. This greennesswellbeing connection has frequently combined built environment with sociodemographic characteristics under the presumption that correlates of subjective wellbeing vary across space and social gradients (Wu et al., 2020b).

Pollution hazards such as the disposal of waste, polluted water, and air pollution increasingly occur at cities from the developing world. This is especially the case in China where spatial disparities of pollution hazards are particularly pronounced, and where decades of urbanization have dramatically raised people's public awareness about subjective wellbeing. Are the perceived pollution hazards a force for mediating the effects of residential greenness on subjective wellbeing, or do they reinforce the diffusion of the greenness-wellbeing connection in the spatial context? Despite intense policy interest in this question, our existing knowledge is limited within a large developing country context. The growing body of empirical literature on the subjective wellbeing evaluation of proximity to green space has so far paid little attention to the role of perceived pollution hazards in influencing the relationship between greenness exposures and subjective wellbeing (Ambrey and Fleming, 2014; Liu et al., 2019b; Xiao et al., 2017).

This paper studies China's eye-sensored green view data to contribute to our understanding of this question. We enrich the literature in twofold. First, we explicitly look at the ways of perceived pollution hazards in confounding the greenness-wellbeing relationship. Previous studies on exploring environmental correlates of subjective wellbeing have mostly focused on the average or population-level effects of proximity to green space (Wu et al., 2019b; Wu et al., 2020b;Xiao et al. 2016). However, individuals may respond to changes in the eye-sensored greenness differently depending on their heterogeneous perceptions about pollution hazards (Cao and Wang, 2016; Lovejoy et al., 2010; Walker, 2011; Wang et al., 2020). For example, a park on which coal dust always falls is not "the same as" a park with a clean environment beside a beautiful river or lake. These environmental amenity differences are likely to be perceived by residents as pollution hazards. Our analysis clarifies the importance of 
conceptualizing the interaction effects of exposures to greenness and perceived pollution hazards on subjective wellbeing. This is consistent with findings from recent studies that have investigated the impacts of objective and/or perceived neighborhood characteristics on subjective wellbeing and health (Cao and Wang, 2016; Elsadek et al., 2019; Foo, 2016; Liu et al., 2019a; Ma et al., 2018b; Vujcic et al., 2019; Xiao et al. 2017; Xiao et al. 2019).If an individual who perceive pollution hazards more heavily than others, he or she may not increase the outdoor green space use even if green views are attractive in his or her neighborhood. Our study points to the policy implication that social and environmental benefits of the provision of urban green infrastructure in promoting people's subjective wellbeing depend on environmental pollution perceptions of local and new residents.

Second, there is a substantial literature dealing with the effect of urban greenness exposures on subjective wellbeing outcomes through geographical measurements of proximity to green space (Ambrey and Fleming, 2014; Fleming et al., 2016) . Much of it is concerned with variation in distance to parks and other types of green amenities within cities, an issue not directly related to our work. Recent studies have moved away from direct geographical measurements that have been widely used over the past decades towards a more explicit measurement about exposure to greenness through deep learning approaches and street view services (Helbich et al., 2019; LeCun et al., 2015). Our study adds to the literature by presenting the empirical assessment that combines sensored street view data with traditional survey data in China to look at the role of perceived pollution hazards in moderating the greenness-wellbeing relationship.

Our assessment carries out in the Beijing metropolitan area because of its increasingly polluted environment as reported by international social media and academic evidence (Kahn and Zheng, 2016). China's rapid economic transformation has accompanied with dramatic changes to the urban landscape and environment, and the decline of subjective wellbeing by residents. Our results provide a basis for policies geared towards accommodating this transformation about the importance of urban greenness in shaping people's lived experiences. This paper is organized as follows: The next section reviews the related literature. Section 3 presents the data and methods. Section 4 discusses the results. Section 5 concludes.

\section{Literature review}

Eye-sensored greenness exposure reflects individuals' eye-senored green views 
the residential environment. It is an important component of environment amenities as perceived by residents' lived experiences. While there is a large number of literature dealing with the subjective wellbeing implications of environmental disamenities such as air pollution within the environmental justice framework (Wolch et al., 2014), recent work has increasingly paid attention on the benefits generated by environmental amenities such as the distribution of urban green space (Lake and Townshend, 2006). Walker (2011) posits that urban greenness represents the alternative topic to be focused in terms of its equitable and just policy outcomes. Understanding the association between eye-sensored greenness exposures and subjective wellbeing has therefore important implications for planners to identify the land use configuration that can improve residents' subjective wellbeing. Indeed, the literature relating greenness to subjective wellbeing has developed rapidly over the past several decades.

Previous studies have shown that urban greenness characteristics are important correlates of subjective wellbeing through providing a restorative context of living environment (Ambrey and Fleming, 2014). Epidemiological evidence has suggested that eye-sensored greenness exposures is helpful for coping with depression and stress in the hectic modern-city life style for live, work and leisure activities (Bowler et al., 2010). The coping mechanisms work partly through the proximity to green amenities at particular places (Gascon et al., 2016), and partly through the quality and levels of greenness as observed by residents (Ord et al., 2013).

Approaches to measure the urban greenness exposures have traditionally been framed within the environmental justice perspective of the proximity regarding about who lives near green space and who does not. Much of it is concerned with the spatial provision of green space and the geographical proximity of green space to residential areas. While most of existing research highlights the subjective wellbeing benefits of living close to green space, empirical results are mixed (Grahn and Stigsdotter, 2010). Recent studies, however, have moved from geographical measurements of distance to parks and green spaces towards explicitly assessing eye-sensored street greenness exposures through big data and deep learning techniques (Helbich et al., 2019). In comparison with geographical distance measurements, eye-sensored greenness views are able to capture street-level vegetation from the 360-degree angles that can be perceived by residents (Wang et al., 2019a).

The presence of urban greenness in the built environment may not always offer good eye-sensored greenness exposures towards people's subjective wellbeing. For 

urban green space when they feel low levels of perceived pollution hazards. Some studies have suggested that perceptions of pollution hazards and safety concerns in a locality may undermine the use of green space and the attractive dimensions of greenness (Walker, 2011). This further points to the implication on subjective wellbeing outcomes generated by perceived neighborhood characteristics. Perceived neighborhood characteristics that reflect individuals' cognitive understandings of objective residential environment can be a mediating channel between objective residential environment and subjective wellbeing outcomes. Lovejoy et al. (2010) suggest that perceived neighborhood characteristics are associated with subjective wellbeing outcomes. Cao (2016) finds that objective built environment characteristics such as density and local amenities significantly influence people's perceived neighborhood characteristics, which in turn contribute to subjective wellbeing. To narrow down the broad inquiries, this study provides a basis for incorporating the perceived pollution hazards characteristics into the evaluation of the association between greenness and subjective wellbeing within a large developing country context. Figure 1 presents the relationship among perceived pollution hazards (air pollution, polluted water, and the disposal of waste), greenness, and subjective wellbeing for illustrating the underlying mechanisms as discussed above. As robustness, we conjecture that residential preferences may be dynamic in nature. On the one hand, residents may adjust their residential preferences and psychological expectations to meet with perceived neighborhood characteristics and become satisfied with where they stay for a long time (Ambrey and Fleming, 2014). On the other hand, residents may relocate to other places if their residential preferences and expectations cannot be fulfilled by the locality (Cao and Wang, 2016). As such we decompose the analysis by stratifying long-term residents and new movers in the neighborhoods, and provide the alternative way to test for the sensitivity of the effects of greenness, perceived pollution hazards and their interaction terms on subjective wellbeing.

\section{Data and Methods}

\subsection{Data}

This study relies on two main datasets. First, we obtain an individual-level survey for people's perceptions about residential environment and socio-demographic 

researchers in 2013 from the Institute of Geographical Sciences and Natural Resources Research, the Chinese Academy of Sciences. A stratified proportional-to-population size sampling technique was applied and questionnaires were circulated to residents in proportion to the population at the neighborhood and district levels based on the recent population census information (Ma et al., 2018b). The survey is designed to be representative of key socio-demographics as compared to the 2010 population census in Beijing. After excluding missing information and data cleaning, 4606 observations distributed in 124 neighborhoods were applied for our study. Residential locations of respondents are geographically coded in the map, on which we can link with urban greenness datasets.

Second, we identify street view images as the main source of urban greenness dataset, which is accessed from the Tencent online mapping services via the API platform (Wang et al., 2019b). Tencent offers one of the most popular social media and mapping services in China, which gives us more confidence on its street view image accuracy. We take three steps in constructing the street view greenness (SVG) index. First, sampling points are set up at the 100 metres distance interval, and are identified along the road network based on OpenStreetMap (Haklay and Weber, 2008). Second, our approach to collect street view images covers $0,90,180$, and 270 degrees relative to each sampling point. Third, we use a combination of the machine learning approach and semantic image segmentation techniques to extract streetscape objects accurately, particularly for trees and grasses (Zhou and Wang, 2019). For each sampling point, the SVG level is measured by the proportion of pixels representing different kinds of green objects as identified in street view images. Figure 2 illustrates the spatial distribution of the eye-sensored greenness exposure levels in the study area.

The variables used in this study include five categories: subjective wellbeing, perceived pollution hazards, individuals' socio-demographics, eye-sensored greenness exposures, and neighbourhood built environment characteristics. The five-category based wellbeing measurement is widely used the science of happiness literature (Fleming et al., 2016; Liu et al., 2019b; Wu et al., 2019b), and is linked with the survey question "How happy are you in your current life conditions?" The survey question is adopted from the mainstream literature and is measured using a five-point Likert-based scale, ranging from "very unhappy" to "very happy". The response of "unknown" has 
been excluded. Our empirical model specification treats this statement as a five-point ordinal variable.

Perceived pollution hazards reflect differences in neighborhood environment as experienced by respondents. We did use the actual pollution levels but rely on perceived pollution hazards for several reasons: First, it is expected that there are limited intracity variations in actual air pollution levels, as compared to inter-city variations. Second, it is difficult to match with actual pollution levels with the time and place of each survey participant due to cross-sectional data limitations. Third, we have rich measures of perceived pollution hazards, which offer an alternative way for sensing lived experiences of pollution exposures to a diverse range of pollution domains. Due to the lack of objective statistics at a fine spatial scale, we focus on the subjective measure of three main perceived pollution hazards - air pollution, polluted water, and the disposal of waste. Regarding perceived air pollution hazards, the survey asked the respondents to make the statement about "how well would you evaluate your experiences about exposure to particulate matter (PM2.5), smog and other air pollution exposures around residential locations?" The survey also asked the respondents about "how well would you evaluate your experiences about rainwater discharge and water pollution around residential locations (namely perceived pollution hazards about polluted water)", and "how well would you evaluate your experiences about pollution from garbage dump and related landfill areas around residential locations (namely perceived pollution hazards about the disposal of waste)." These statements are evaluated by using a fivepoint Likert-based scale, ranging from "very well" to "very poor".

The survey has reported a list of sociodemographic characteristics including age, gender, educational attainment levels, employment status, income, homeownership status, residence status (local residents in the host city versus migrants) and so on. Further, respondents reported about whether they have experienced residential relocations over the past five years. This reported statement provides us the clear evidence on stratifying residents into movers and non-movers social groups, on which we can test for the residential preference concern (Cao and Wang, 2016). Following the literature (Ambrey and Fleming, 2014; Cao, 2016; Wu et al., 2019a), the sociodemographic variables such as Gender、Hukou、Mover、Homeownership are constructed as binary indicators directly based on the survey questions. To simplify the analysis, we categorize age below and above 40 based on the sample median age in the 
survey data. The richness of survey information offers us the opportunity to control for key observable individual sociodemographic characteristics in the model specifications that may confound the results.

In terms of the eye-sensored greenness exposures, we measure the SVG level by averaging the SVG scores for all sampling points within 1000-metre circular buffers relative to each respondent's residential location. Neighborhood (jie dao) refers to the fundamental census administration unit in Beijing, with the average size of 12 squared kilometers in our study. Ideally it would be meaningful to control for the building-block or community-level characteristics as suggested by studies in developed countries (Ambrey and Fleming, 2014; Houlden et al., 2019). However, there lacks the finerscale census information for illustrating local area sociodemographic in Chinese cities (Ma et al., 2018a). We acknowledge this limitation. Neighborhood built environment characteristics such as population density, proportion of historical buildings built before 1949 , the ratio of the total aboveground floor areas relative to the neighbourhood land areas (plot ratio, thereafter), and proximity to local amenities are controlled in the empirical model specifications. Table 1 reports the descriptive statistics of variables.

\subsection{Methods}

Our aim is to examine the influences of eye-sensored greenness exposures, perceived pollution hazards, and their interaction terms on subjective wellbeing. As the subjective wellbeing outcome is measured on an ordinal scale, we employ ordered response models to carry out the estimation. Methodologically, we consider the multiple spatial levels of our data structure, where respondents are located in neighbourhoods. This multi-level data context implies that residents who live in neighbouring places may have experienced particular domains of pollution hazards in a similar pattern due to the presence of spatial dependency. The existing literature on the evaluation of individual survey data has increasingly paid attention to the role of spatial effects in the analysis (Ma et al., 2018b; Wu and Hong, 2017). As such our modelling approach uses the Bayesian multilevel ordinal response model (Goldstein, 2010) through the following logit link function:

$$
\begin{array}{r}
\log \left(\frac{P\left(y_{i n, t} \leqslant t\right)}{1-P\left(y_{i n, t} \leqslant t\right)}\right)=\alpha_{t}+\left(\beta+\mu_{g n}\right) \text { Greenness }+\left(\gamma+\mu_{p n}\right) \text { Pollution }+ \\
\eta \text { Greenness } \cdot \text { Pollution }+\varphi \boldsymbol{X}_{i n}^{\prime}+\psi \boldsymbol{Z}_{n}^{\prime}+\mu_{0 n}+\epsilon_{0 \text { in }}
\end{array}
$$


Where $y_{i n, t}$ represent the subjective wellbeing level of individual $i$ who live in neighbouring places $n . \quad P\left(y_{i n, t} \leqslant t\right)$ represents the cumulative probability of the score falling in the $t$-th category or below. $\alpha_{t}$ is the intercept term associated with the cumulative distribution of the $t$-th response category at neighbourhood level. Greenness and Pollution are urban greenness and the perceived pollution hazards(the disposal of waste, polluted water, and air pollution), respectively. Greenness.Pollution is the interaction term of urban greenness and the perceived pollution hazards. Considering that the slope of the explanatory variables varies randomly across neighbourhoods, the random terms $\mu_{g n}$ and $\mu_{p n}$ are added to the coefficient of Greenness $(\beta)$ and Pollution $(\gamma) . \boldsymbol{X}_{\text {in }}^{\prime}$ is a vector of socio-demographic covariates, such as age, gender, educational attainment level, employment status, income, homeownership and so on. $\boldsymbol{Z}_{n}^{\prime}$ is a vector of neighbourhood(jiedao)-level covariates, including distance to central business district(CBD) and subway from each neighbourhood, plot ratio, total population density, and proportion of historical buildings built before 1949 in each neighbourhood.

$$
\begin{gathered}
{\left[\begin{array}{l}
\mu_{0 n} \\
\mu_{g n}
\end{array}\right] \sim N\left(0, \Omega_{\mu 1}\right),\left[\begin{array}{l}
\mu_{0 n} \\
\mu_{p n}
\end{array}\right] \sim N\left(0, \Omega_{\mu 2}\right) ;} \\
\Omega_{\mu 1}=\left[\begin{array}{ll}
\sigma_{\mu 0}^{2} & \\
\sigma_{\mu 0 g} & \sigma_{\mu g}^{2}
\end{array}\right], \quad \Omega_{\mu 2}=\left[\begin{array}{ll}
\sigma_{\mu 0}^{2} & \\
\sigma_{\mu 0 p} & \sigma_{\mu p}^{2}
\end{array}\right], \quad \epsilon_{0 i n} \sim N\left(0, \sigma_{\epsilon 0}^{2}\right)
\end{gathered}
$$

The Bayesian multilevel ordered logit model captures the spatial dependence effects of multi-dimensional spatial scales by dismantling the total variance into variation between, and variation within the neighbourhood level units (Goldstein et al., 2002). In our paper, the variance of the outcome term is divided into two components, $\sigma_{\mu 0}^{2}$ and $\sigma_{\epsilon 0}^{2}$.The variance in intercepts between neighbourhoods $\left(\sigma_{\mu 0}^{2}\right)$ captures unobservable between-neighbourhood variability, and the variance in intercepts within neighbourhoods $\left(\sigma_{\epsilon 0}^{2}\right)$ is assumed to be fixed across different neighbourhoods which captures unobservable within-neighbourhood variability. In addition, $\sigma_{u g}^{2}$ and $\sigma_{u p}^{2}$ in the random slope model are the variances in slopes between neighbourhoods. $\sigma_{\mu 0 g}$ and $\sigma_{\mu 0 p}$ are the covariances between intercepts and slopes. Furthermore, the Bayesian multilevel ordered logit model based on the Markov Chain Monte Carlo (MCMC) Bayesian estimation, because commonly used methods such as maximum likelihood estimation have a highly unstable in the estimation of variance parameters (Ma et al., 2018b). 


\section{Results}

\subsection{Main results}

Table 2 reports the main results by using the Bayesian multilevel ordered logit model. Columns (1)-(3) presents different model specifications with the inclusion of perceived pollution hazards about the disposal of waste, polluted water, and air pollution respectively. We note that the coefficient is at the 0.05 level of significance and better when the zero value is not included in the $95 \%$ credible interval (CI) range.

We find that eye-sensored greenness exposure is positively associated with subjective wellbeing at the conventional significance level. To simplify the interpretation of the results, we use the decentralized procedure (Jaccard et al., 1990) to standardize key coefficients with respect to greenness and perceived pollution hazards. The probability of being satisfied increase by about 2.77 times [exp(1.019)] if being exposed to higher greenness levels. Estimates from columns (2) and (3) are of similar magnitudes, and provide additional evidence in favor of the positive effects of greenness on subjective wellbeing. These results are largely consistent with findings from recent epidemiological studies (Bowler et al., 2010). The possible mechanism lies in the levels of eye-sensored street greenness exposure (Ord et al., 2013). The influence of perceived pollution hazards domains on subjective wellbeing is complicated. To begin with, we find that all of the perceived pollution hazards domains are significant correlates with subjective wellbeing. The probability of being satisfied increase by about 1.03 times [1/exp(-0.331)] if being exposed to lower waste pollution levels, and the corresponding quantified results from columns (2) and (3) are about 1.38 times [1/exp(-0.320)] and 1.22 times [1/exp(-0.200)], respectively. Respondents who perceive lower pollution hazards about air pollution, polluted water and the disposal of waste are more likely to report better subjective wellbeing status.

After considering interaction terms, our results suggest the significant role of perceived pollution hazards about the disposal of waste, polluted water, and air pollution in influencing the association between eye-sensored greenness exposures and subjective wellbeing. We find that the positive magnitudes of the greenness-subjective wellbeing relationship tend to be shrink when considering perceived pollution hazards about the disposal of waste, polluted water, and air pollution. The plotted patterns from Figure 3 illustrate the distributional effects from greenness and perceived pollution hazards on subjective wellbeing when moving from people who are less happy $(=1)$ to 
people who are very happy (=5). The significant signs are judged by if the CI range is crossed-over with the zero. We find that the effects of greenness exposures on subjective wellbeing mainly come from those who are less satisfied about pollution hazards. The insignificant sign associated with the interaction term of perceived pollution hazards about air pollution and greenness can be partly explained by the observation that there are not markedly differences in air pollution levels over space within a city as compared to inter-city variations in air pollution levels. It is in this sense that residents may not be quite sensitive to the interaction of greenness and perceptions about air pollution across urban neighbourhoods. We proceed with two robustness checks. First, we have re-run the models in Table 2 without the interaction term of greenness*pollution and the main results remain robust. Second, the survey has the respondents' perception about parks, green space and green belts, which can be loosely regarded as the perceived neighbourhood greenness. The inclusion of this indicator into Table 2 is significantly correlated with subjective wellbeing, but did not affect the significance of other key variables. The robustness results are not tabulated.

In terms of demographics, our results are consistent with recent findings from the literature suggesting that income, employment, education and homeownership status are significantly associated with subjective wellbeing. Most of demographic variables make sense. For example, respondents who have local hukou, higher income levels, and higher educational attainment levels tend to report better subjective wellbeing status. Turning into built environment characteristics, respondents tend to be more satisfied when they live in dense neighbourhoods with close proximity to CBD and subway. People are less satisfied in neighbourhoods with high proportions of historical buildings built before 1949, probably due to the lack of well-serviced communities (Huang et al., 2020).

\subsection{Heterogeneous effects across social and spatial gradients}

We report on a set of sensitivity analyses to show the heterogenous effects across social and spatial gradients. First, our main results have concentrated on the interaction effects of eye-sensored greenness exposures and perceived pollution hazards on subjective wellbeing for the whole sampled residents without considering residential preferences that may be dynamic over time. To partly test for this concern, Table 3 reports the results by comparing the sub-samples of movers and non-movers. As expected, we find that movers and non-movers have different patterns on correlates of 
subjective wellbeing. The third row in Panel A and Panel B reports the estimates of the interaction term between eye-sensored greenness exposures and perceived pollution hazards that are of interest to this analysis. Results from columns (1)-(3) suggest that there are markedly differences between movers and non-movers in the greennesspollution interaction effects when interacting with waste and air pollution dimensions. These differentiated findings are reasonable since non-movers are long-term stayers in the neighbourhoods as compared to movers. It is likely that non-movers may have adopted their needs and expectations to fit with residential environment and have got used to pollution hazards since they have lived in the neighbourhood for more than five years. However, movers' residential preferences are likely to be sensitive to pollution hazards since they have recently selected themselves into the current places to live. Our models are unable to fully capture changes in people's perceptions due to the lack of individual panel data. But as a baseline these findings imply that using green space planning policies to promote subjective wellbeing may have limited effects without considering the dynamic residential preferences channel at work.

Second, we assess the robustness of the main results by decomposing the effects across social and spatial gradients. Table 4 explores heterogeneity in our baseline estimates by stratifying individuals and neighbourhoods across key observable characteristics. Each row represents a separate model specification. In terms of social gradients, the individual characteristics we explore are educational attainment level, age, and employment status. For spatial gradients, we group neighbourhoods according to the median value of a particular variable of interest. We consider the differences in population density and the proportion of historical buildings that may mask the heterogeneity in the results.

Table 4 shows that the interaction effects of eye-sensored greenness exposures and perceived pollution hazards on subjective wellbeing is larger for relatively young people and higher educated social groups. One potential explanation is that, residents with better education attainment levels are more likely to recognize about the influences of perceived pollution hazards and greenness on their subjective wellbeing levels. In addition, there is evidence that residents who are full time workers have a more pronounced interaction effects on subjective wellbeing than those without full time employment status. Turning to the spatial gradients, the estimates suggest that the interaction effects on subjective wellbeing respond more to neighbourhoods with low population density and high proportions of historical buildings. In addition to subjective 
wellbeing implication of these estimates, it is noteworthy that if residents are aware of these pollution hazards measures and they are perceived negatively by residents, one would have expected to see this further capitalized in differences in housing values. The point estimates are not distributed evenly across different domains of perceived pollution hazards, though these decomposing analyses are not quite robust for definitive conclusions. Together, the heterogeneous pattern across neighbourhoods support the environmental justice concern for residents perceiving inequality lived experiences towards eye-sensored greenness exposures.

In the last two rows, we present two additional robustness checks. First, we present estimates for the variations in how we define the distance buffers in measuring the spatial range of eye-sensored greenness exposures. We use greenness exposures at the 500-meter distance buffer relative to residential locations, instead of a 1000-meter distance buffer. This results in significant estimates with smaller magnitudes, suggesting that the interaction effects are distributed in a spatially non-linear matter. Second, we use the traditional ordered logit model for comparison. The results remain largely robust in terms of qualitative nature but turns to be less significant. This implies the importance of considering spatial multilevel effects into the evaluation for mitigating the biased statistical inference concern. It is also important to note that the Bayesian estimation fits the data better than traditional models (Wu and Hong, 2017) as evidenced by the Deviance Information Criterion (DIC) values. Taken together, there is strong evidence on how the interaction effects of eye-sensored greenness exposures and perceived pollution hazards vary with social and spatial gradients.

\section{Conclusions}

This study examines whether perceived pollution hazards have an interaction effect with eye-sensored greenness exposures on subjective wellbeing in using a combination of individual survey and greenness datasets from Beijing, China. Our analysis enriches the debate in the literature of the relationships among urban greenness, perceived neighbourhood characteristics, and subjective wellbeing within a large developing country context, where pollution hazards are highly sensitive issues (Kahn and Zheng, 2016).

Our results suggest that eye-sensored greenness exposure significantly contributes to subjective wellbeing for residents in the ways that are consistent with the literature: residents being exposure to higher greenness are happier with life, after controlling for 
individual socio-demographic characteristics and built environment characteristics (Liu et al., 2019b; Wang et al., 2020). Our analysis supports the findings from recent studies (Chen et al., 2013; Peek et al., 2009) suggesting the significant influences of perceived pollution hazards on subjective health and wellbeing. We find that perceived pollution hazards about the disposal of waste, polluted water, and air pollution have significant interaction effects on moderating the greenness-subjective wellbeing relationship. These findings suggest that perceived pollution hazards and eye-sensored greenness exposures have the complementary role to play in influencing subjective wellbeing. As suggested by recent studies in green space contexts (Dong and Qin, 2017; Wu et al., 2019b; Wu et al., 2020a) such complementary effects are not distributed evenly across social and spatial gradients.

Our results provide implications on a wave of potentially large infrastructure improvements for promoting urban greenness in the built environment with health and wellbeing initiatives (Lachowycz and Jones, 2013). In the context of planning to improve the capacities of green amenities and thereby enhance people's subjective wellbeing, the perception of pollution hazards responds to a range of lived experiences and anticipations may be constrained by use frequencies (Wu et al., 2020b) and spacetime scales (Schwanen and Wang, 2014). By considering intervention toolkits flowing from pollution hazards to other perceived neighbourhood characteristics, planners can subsequently establish effective policy interventions and landscape designs for enhancing the provision of urban greenness to a wide array of users. Our analytical framework can help move the impetus of land use planning from the traditional normative wisdom with reducing the distributional inequality of green space towards the perception-contextualized dependent nature of neighbourhood environment (Lovejoy et al., 2010). We did not adopt traditional geographical distance-based proximity measures but rather eye-sensored street view greenness coded from online mapping services. We did not deny the importance of proximity to green space, but instead focused on the complementary effects of perceived neighbourhood characteristics with eye-sensored greenness exposures through careful examination of what people perceive in their lived experiences about pollution hazards. The emphasis on pollution hazards embedded in our framework refocuses the planning agenda for the urban green space provision from stressing proximity to developing a more complete understanding for exposures as offered by environmental hazards (Ma et al., 2016). In this sense the presented findings provide the prospect of recalibrating the green 


\section{References}

Ambrey, C., Fleming, C., 2014. Public Greenspace and Life Satisfaction in Urban Australia. Urban Studies 51, 1290-1321. https://doi.org/10.1177/0042098013494417

Bowler, D.E., Buyung-Ali, L.M., Knight, T.M., Pullin, A.S., 2010. A systematic review of evidence for the added benefits to health of exposure to natural environments. BMC Public Health 10, 456. https://doi.org/10.1186/1471-2458$10-456$

Cao, X. (Jason), 2016. How does neighborhood design affect life satisfaction? Evidence from Twin Cities. Travel Behaviour and Society 5, 68-76. https://doi.org/https://doi.org/10.1016/j.tbs.2015.07.001

Cao, X. (Jason), Wang, D., 2016. Environmental correlates of residential satisfaction: An exploration of mismatched neighborhood characteristics in the Twin Cities. Landscape and Urban Planning 150, 26-35. https://doi.org/10.1016/j.landurbplan.2016.02.007

Chen, J., Chen, S., Landry, P.F., 2013. Migration, environmental hazards, and health outcomes in China. Social Science and Medicine 80, 85-95. https://doi.org/10.1016/j.socscimed.2012.12.002

Dong, H., Qin, B., 2017. Exploring the link between neighborhood environment and mental wellbeing: A case study in Beijing, China. Landscape and Urban Planning 164, 71-80. https://doi.org/10.1016/j.landurbplan.2017.04.005

Elsadek, M., Liu, B., Lian, Z., 2019. Green façades : Their contribution to stress recovery and well-being in high-density cities. Urban Forestry \& Urban Greening 46, 126446. https://doi.org/10.1016/j.ufug.2019.126446

Fleming, C.M., Manning, M., Ambrey, C.L., 2016. Crime, greenspace and life satisfaction: An evaluation of the New Zealand experience. Landscape and Urban Planning 149, 1-10. https://doi.org/10.1016/j.landurbplan.2015.12.014

Foo, C.H., 2016. Linking forest naturalness and human wellbeing - A study on public's experiential connection to remnant forests within a highly urbanized region in Malaysia. Urban Forestry \& Urban Greening 16, 13-24. https://doi.org/10.1016/j.ufug.2016.01.005

Gascon, M., Triguero-Mas, M., Martínez, D., Dadvand, P., Rojas-Rueda, D., Plasència, A., Nieuwenhuijsen, M.J., 2016. Residential green spaces and mortality: A systematic review. Environment International 86, 60-67. https://doi.org/https://doi.org/10.1016/j.envint.2015.10.013

Goldstein, H., 2010. Multilevel Statistical Models(4th Edition). John Wiley \& Sons, Ltd 15-72. https://doi.org/10.1002/9780470973394

Goldstein, H., Browne, W., Rasbash, J., 2002. Partitioning Variation in Multilevel Models. Understanding Statistics 1, 223-231. https://doi.org/10.1207/s15328031us0104_02

Grahn, P., Stigsdotter, U.K., 2010. The relation between perceived sensory 
dimensions of urban green space and stress restoration. Landscape and Urban Planning 94, 264-275. https://doi.org/https://doi.org/10.1016/j.landurbplan.2009.10.012

Haklay, M., Weber, P., 2008. OpenStreetMap: User-Generated Street Maps. IEEE Pervasive Computing 7, 12-18. https://doi.org/10.1109/MPRV.2008.80

Helbich, M., Yao, Y., Liu, Y., Zhang, J., Liu, P., Wang, R., 2019. Using deep learning to examine street view green and blue spaces and their associations with geriatric depression in Beijing, China. Environment International 126, 107-117. https://doi.org/https://doi.org/10.1016/j.envint.2019.02.013

Houlden, V., Porto de Albuquerque, J., Weich, S., Jarvis, S., 2019. A spatial analysis of proximate greenspace and mental wellbeing in London. Applied Geography 109, 102036. https://doi.org/10.1016/j.apgeog.2019.102036

Huang, L., Zheng, W., Hong, J., Liu, Y., Liu, G., 2020. Paths and strategies for sustainable urban renewal at the neighbourhood level: A framework for decisionmaking. Sustainable Cities and Society 55, 102074. https://doi.org/10.1016/j.scs.2020.102074

Jaccard, J., Wan, C.K., Turrisi, R., 1990. The Detection and Interpretation of Interaction Effects Between Continuous Variables in Multiple Regression. Multivariate Behavioral Research 25, 467-478. https://doi.org/10.1207/s15327906mbr2504_4

Kahn, M.E., Zheng, S., 2016. Blue Skies over Beijing: Economic Growth and the Environment in China. Princeton. NJ: Princeton University Press 1-288. https://doi.org/10.7312/hart91728-003

Kwan, M.P., 2012. Geographies of Health. Annals of the Association of American Geographers 102, 891-892. https://doi.org/10.1080/00045608.2012.687348

Lachowycz, K., Jones, A.P., 2013. Towards a better understanding of the relationship between greenspace and health: Development of a theoretical framework. Landscape and Urban Planning 118, 62-69. https://doi.org/https://doi.org/10.1016/j.landurbplan.2012.10.012

Lake, A., Townshend, T., 2006. Obesogenic Environments: Exploring the Built and Food Environments. The journal of the Royal Society for the Promotion of Health 126, 262-267. https://doi.org/10.1177/1466424006070487

LeCun, Y., Bengio, Y., Hinton, G., 2015. Deep learning. Nature 521, 436-444. https://doi.org/10.1038/nature14539

Lindholst, A., Dempsey, N., Burton, M., 2013. Introducing a method for mapping recreational experience. Urban Design and Planning 166, 296-308. https://doi.org/10.1680/udap.11.00012

Liu, Y., Wang, R., Xiao, Y., Huang, B., Chen, H., Li, Z., 2019a. Exploring the linkage between greenness exposure and depression among Chinese people: Mediating roles of physical activity, stress and social cohesion and moderating role of urbanicity. Health and Place 58, 102168. 
https://doi.org/10.1016/j.healthplace.2019.102168

Liu, Ye, Wang, R., Grekousis, G., Liu, Yuqi, Yuan, Y., Li, Z., 2019b. Neighbourhood greenness and mental wellbeing in Guangzhou, China: What are the pathways? Landscape and Urban Planning 190, 103602. https://doi.org/10.1016/j.landurbplan.2019.103602

Lovejoy, K., Handy, S., Mokhtarian, P., 2010. Neighborhood satisfaction in suburban versus traditional environments: An evaluation of contributing characteristics in eight California neighborhoods. Landscape and Urban Planning 97, 37-48. https://doi.org/https://doi.org/10.1016/j.landurbplan.2010.04.010

Ma, J., Chen, Y., Dong, G., 2018a. Flexible Spatial Multilevel Modeling of Neighborhood Satisfaction in Beijing. Professional Geographer 70, 11-21. https://doi.org/10.1080/00330124.2017.1298453

Ma, J., Dong, G., Chen, Y., Zhang, W., 2018b. Does satisfactory neighbourhood environment lead to a satisfying life? An investigation of the association between neighbourhood environment and life satisfaction in Beijing. Cities 74, 229-239.

Ma, J., Mitchell, G., Dong, G., Zhang, W., 2016. Inequality in Beijing: A Spatial Multilevel Analysis of Perceived Environmental Hazard and Self-Rated Health. Annals of the American Association of Geographers 107, 109-129. https://doi.org/10.1080/24694452.2016.1224636

Ord, K., Mitchell, R., Pearce, J., 2013. Is level of neighbourhood green space associated with physical activity in green space? International Journal of Behavioral Nutrition and Physical Activity 10, 127. https://doi.org/10.1186/1479-5868-10-127

Peek, M.K., Cutchin, M.P., Freeman, D., Stowe, R.P., Goodwin, J.S., 2009. Environmental hazards and stress: Evidence from the Texas City Stress and Health Study. Journal of Epidemiology and Community Health 63, 792-798. https://doi.org/10.1136/jech.2008.079806

Schwanen, T., Wang, D., 2014. Well-Being, Context, and Everyday Activities in Space and Time. Annals of the Association of American Geographers 104, 833851. https://doi.org/10.1038/nrn2891

Vujcic, M., Tomicevic-dubljevic, J., Zivojinovic, I., Toskovic, O., 2019. Connection between urban green areas and visitors ' physical and mental. Urban Forestry \& Urban Greening 40, 299-307. https://doi.org/10.1016/j.ufug.2018.01.028

Walker, G., 2011. Environmental justice: Concepts, evidence and politics. Routledge 1edition, 1-272. https://doi.org/10.4324/9780203610671

Wang, R., Helbich, M., Yao, Y., Zhang, J., Liu, P., Yuan, Y., Liu, Y., 2019a. Urban greenery and mental wellbeing in adults: Cross-sectional mediation analyses on multiple pathways across different greenery measures. Environmental Research 176, 108535. https://doi.org/https://doi.org/10.1016/j.envres.2019.108535

Wang, R., Liu, Y., Lu, Y., Zhang, J., Liu, P., Yao, Y., Grekousis, G., 2019 b. 
Perceptions of built environment and health outcomes for older Chinese in Beijing: A big data approach with street view images and deep learning technique. Computers, Environment and Urban Systems 78, 101386. https://doi.org/https://doi.org/10.1016/j.compenvurbsys.2019.101386

Wang, R., Yang, B., Yao, Y., Bloom, M.S., Feng, Z., Yuan, Y., Zhang, J., Liu, P., Wu, W., Lu, Y., Baranyi, G., Wu, R., Liu, Y., Dong, G., 2020. Residential greenness, air pollution and psychological well-being among urban residents in Guangzhou, China. Science of the Total Environment 711, 134843. https://doi.org/10.1016/j.scitotenv.2019.134843

Wolch, J.R., Byrne, J., Newell, J.P., 2014. Urban green space, public health, and environmental justice: The challenge of making cities 'just green enough.' Landscape and Urban Planning 125, 234-244. https://doi.org/https://doi.org/10.1016/j.landurbplan.2014.01.017

Wu, W., Chen, Y., Ye, L., 2020a. Perceived spillover effects of club-based green space : Evidence from Beijing golf courses , China. Urban Forestry \& Urban Greening 48, 126518. https://doi.org/10.1016/j.ufug.2019.126518

Wu, W., Dong, G., SUN, Y., Yun, Y., 2020b. Contextualized effects of Park access and usage on residential satisfaction: A spatial approach. Land Use Policy 94, 104532. https://doi.org/10.1016/j.landusepol.2020.104532

Wu, W., Hong, J., 2017. Does public transit improvement affect commuting behavior in Beijing, China? A spatial multilevel approach. Transportation Research Part D: Transport and Environment 52, 471-479. https://doi.org/https://doi.org/10.1016/j.trd.2016.08.032

Wu, W., Stephens, M., Du, M., Wang, B., 2019a. Homeownership, family composition and subjective wellbeing. Cities 84, 46-55. https://doi.org/10.1016/j.cities.2018.07.004

Wu, W., Xin, M., Zhu, N., Zhang, W., Sun, H., 2019b. Residential satisfaction about urban greenness : Heterogeneous effects across social and spatial gradients. Urban Forestry \& Urban Greening 38, 133-144. https://doi.org/10.1016/j.ufug.2018.11.011

Xiao, Y., Wang, D. and Fang, J. (2019). Exploring the disparities in park access through mobile phone data: Evidence from Shanghai, China. Landscape and Urban Planning, 181(C), 80-91.

Xiao, Y., Wang, Z., Li, Z., \& Tang Z. (2017). An assessment of urban park access in Shanghai - Implications for the social equity in urban China. Landscape and Urban Planning, DOI: 10.1016/j.landurbplan.2016.08.007

Xiao, Y., Li, Z., \& Webster, C. (2016). Estimating the mediate effect of privately green space on the relationship between urban public green space and property value: Evidence from Shanghai, China. Land Use Policy, 54, 439-447.

Zhou, M., Wang, D., 2019. Generational differences in attitudes towards car , car ownership and car use in Beijing. Transportation Research Part D 72, 261-278. https://doi.org/10.1016/j.trd.2019.05.008 


\section{Figure list}

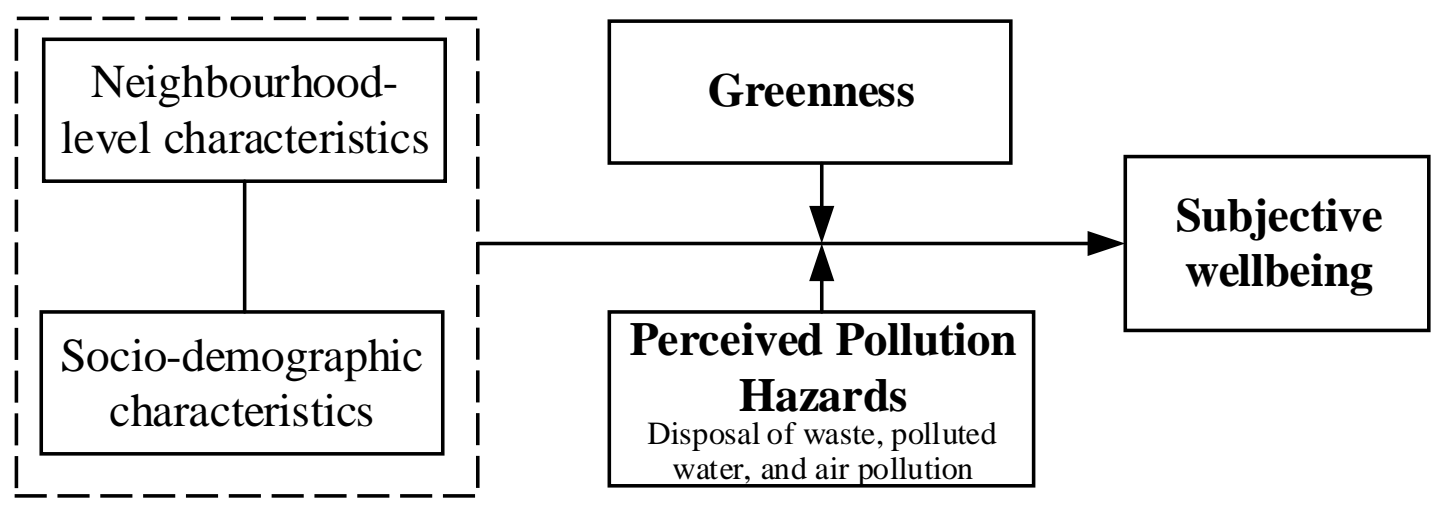

Figure 1. Conceptual framework 
1

2

3

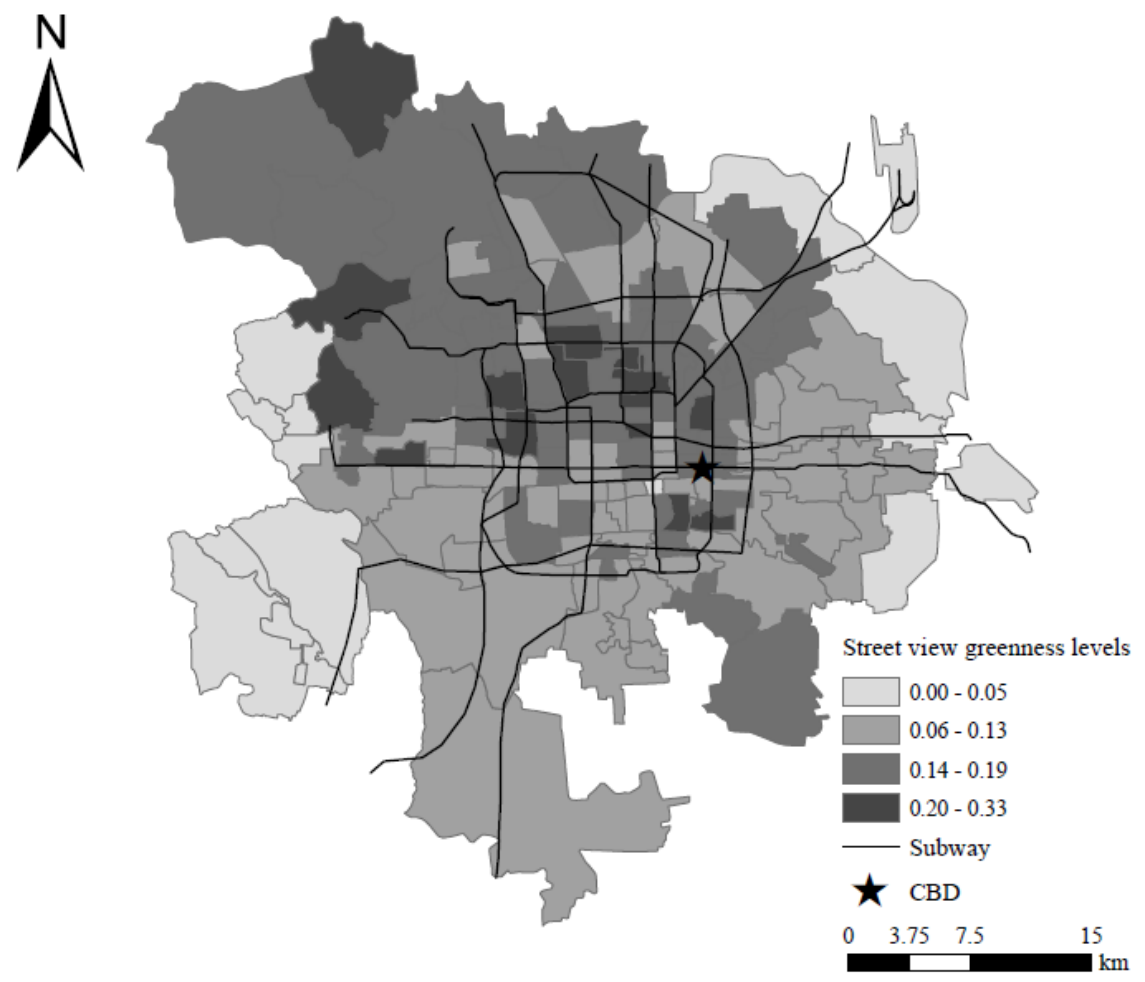

Figure 2. The distributions of the street view greenness(SVG) level, subway, and central business district(CBD) in the study area.

Notes: The street view greenness(SVG) level was classified base d on natural break rule. 

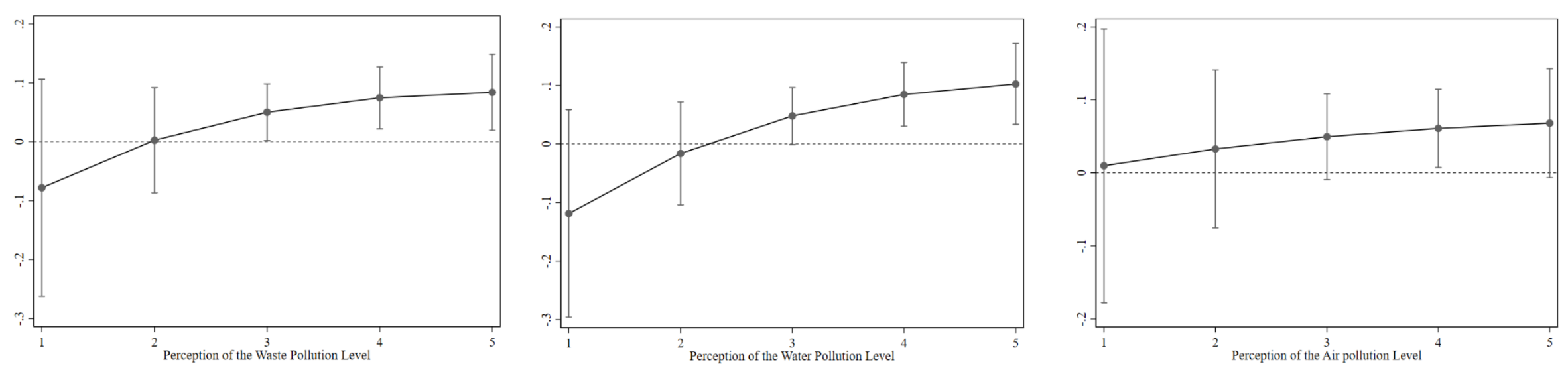

Figure 3. Distributional effects from greenness and perceived pollution hazards on subjective wellbeing with the 95\%CI range

Notes: The X-axis from the left figure to the right figure is the perception of the disposal of waste, polluted water, and air pollution, respectively.

The Y-axis shows the marginal effect on subjective wellbeing. 


\section{Table list}

Table 1. Descriptive statistics

\begin{tabular}{|c|c|c|}
\hline Variable & Description & $\begin{array}{c}\text { Proportion/mean } \\
(\mathrm{SD})\end{array}$ \\
\hline Subjective wellbeing & $\begin{array}{l}\text { How happy are you in your current life conditions(1-5 } \\
\text { denotes "very unhappy" to "very happy") }\end{array}$ & $\begin{array}{c}\text { Very happy }(5.76 \%) \\
\text { Happy }(49.08 \%) \\
\text { Neutral }(39.46 \%) \\
\text { Unhappy }(5.20 \%) \\
\text { Very unhappy }(0.50 \%)\end{array}$ \\
\hline Greenness & $\begin{array}{l}\text { Street view greenness (SVG) within a } 1 \mathrm{~km} \text { buffer around } \\
\text { residential location }\end{array}$ & $0.13(0.065)$ \\
\hline Waste pollution & $\begin{array}{l}\text { Perception about pollution from garbage dump and related } \\
\text { landfill areas around residential locations (1-5 denotes } \\
\text { "very well" to "very poor") }\end{array}$ & 2.95(0.920) \\
\hline Water pollution & $\begin{array}{l}\text { Perception about rainwater discharge and water pollution } \\
\text { around residential locations (1-5 denotes "very well" to } \\
\text { "very poor") }\end{array}$ & 2.97(0.927) \\
\hline Air pollution & $\begin{array}{l}\text { Perception about particulate matter (PM2.5), smog and } \\
\text { other air pollution exposures ( } 1-5 \text { denotes "very well" to } \\
\text { "very poor") }\end{array}$ & $3.45(0.927)$ \\
\hline \multicolumn{3}{|c|}{ Socio-demographic covariates } \\
\hline Age & Binary variable: age above 40 as the reference category & $72.82 \%$ \\
\hline Gender & Binary variable: female as the reference category & $50.83 \%$ \\
\hline Education & $\begin{array}{l}\text { Binary variable: high school level and below as reference } \\
\text { category }\end{array}$ & $63.76 \%$ \\
\hline Employment status & Binary variable: non-full time worker as reference category & $85.78 \%$ \\
\hline Mover & Living in the current residence for less than 5 years & $26.07 \%$ \\
\hline Hukou & Binary variable: non-local hukou as reference category & $65.26 \%$ \\
\hline Income(below 5000) & Monthly income below 5000 RMB & $27.64 \%$ \\
\hline Income(5000-9999) & Monthly income between 5000 and 9999 RMB & $34.64 \%$ \\
\hline $\begin{array}{l}\text { Income }(10000- \\
15000)\end{array}$ & Monthly income between 10000 and 15000 RMB & $21.24 \%$ \\
\hline $\begin{array}{l}\text { Income(above } \\
15000)\end{array}$ & Monthly income above $15000 \mathrm{RMB}$ & $16.48 \%$ \\
\hline Homeownership & Binary variable: renter as reference category & $51.61 \%$ \\
\hline \multicolumn{3}{|c|}{ Neighborhood covariates } \\
\hline Distance to CBD & Distance to the central business district in kilometers & $11.57(6.094)$ \\
\hline Distance to subway & Distance to the nearest subway in kilometers & $0.88(1.177)$ \\
\hline Plot ratio & $\begin{array}{l}\text { The ratio of the total aboveground floor area to the land } \\
\text { area of a neighborhood }\end{array}$ & $0.88(0.522)$ \\
\hline Population density & $\begin{array}{l}\ln (\text { Total population density in each neighborhood (persons } \\
\left.\text { per } \mathrm{km}^{2}\right) \text { ) }\end{array}$ & $1.91(2.780)$ \\
\hline Heritage architecture & $\begin{array}{l}\text { Proportion of historical buildings built before } 1949 \text { in each } \\
\text { neighborhood }\end{array}$ & $0.01(0.016)$ \\
\hline
\end{tabular}


Table 2. Baseline results

\begin{tabular}{|c|c|c|c|}
\hline & (1) & (2) & (3) \\
\hline Greenness & $1.019 *(0.943-1.097)$ & $1.037 *(0.939-1.125)$ & $1.101 *(0.982-1.217)$ \\
\hline Pollution & $-0.331 *(-0.387--0.271)$ & $-0.320 *(-0.398--0.240)$ & $-0.200 *(-0.278--0.122)$ \\
\hline Greenness*Pollution & $0.775 *(0.631-0.917)$ & $0.981 *(0.843-1.143)$ & $0.583 *(0.487-0.684)$ \\
\hline Age & $0.044(-0.019-0.117)$ & $0.037(-0.057-0.126)$ & $0.009(-0.121-0.117)$ \\
\hline Gender & $-0.019(-0.093-0.053)$ & $-0.029(-0.103-0.057)$ & $-0.030(-0.121-0.067)$ \\
\hline Education & $0.110 *(0.024-0.204)$ & $0.131 *(0.036-0.222)$ & $0.085(-0.037-0.207)$ \\
\hline Employment status & $-0.286 *(-0.372--0.202)$ & $-0.326 *(-0.391--0.254)$ & $-0.289 *(-0.331--0.240)$ \\
\hline Hukou & $0.190 *(0.096-0.283)$ & $0.197 *(0.116-0.279)$ & $0.205 *(0.093-0.316)$ \\
\hline Income(5000-9999) & $0.223 *(0.092-0.357)$ & $0.288 *(0.205-0.369)$ & $0.334 *(0.276-0.383)$ \\
\hline Income $(10,000-15,000)$ & $0.329 *(0.197-0.458)$ & $0.401 *(0.313-0.484)$ & $0.428 *(0.360-0.497)$ \\
\hline Income(above 15,000$)$ & $0.602 *(0.520-0.692)$ & $0.674 *(0.637-0.723)$ & $0.747 *(0.609-0.882)$ \\
\hline Homeownership & $0.359 *(0.289-0.427)$ & $0.327 *(0.229-0.421)$ & $0.249 *(0.163-0.345)$ \\
\hline Mover & $0.103 *(0.040-0.164)$ & $0.060(-0.059-0.160)$ & $0.087 *(0.012-0.159)$ \\
\hline Distance to the CBD & $-0.012(-0.026-0.003)$ & $-0.015^{*}(-0.025--0.004)$ & $-0.011(-0.027-0.005)$ \\
\hline Distance to the nearest subway & $-0.046 *(-0.080--0.009)$ & $-0.054(-0.112-0.002)$ & $-0.058(-0.121-0.002)$ \\
\hline Plot ratio & $-0.055(-0.136-0.027)$ & $-0.049(-0.187-0.089)$ & $0.026(-0.102-0.152)$ \\
\hline Population density & $0.011(-0.014-0.038)$ & $0.005(-0.019-0.030)$ & $0.009(-0.016-0.035)$ \\
\hline Heritage architecture & $-1.089 *(-1.157--1.016)$ & $-0.978 *(-1.162--0.790)$ & $-1.202 *(-1.320--1.054)$ \\
\hline$\sigma_{\mu 0}^{2}$ & $0.147 *(0.084-0.237)$ & $0.161 *(0.090-0.255)$ & $0.140 *(0.080-0.221)$ \\
\hline$\sigma_{u g}^{2}$ & $1.317 *(0.045-6.721)$ & $0.162 *(0.006-0.597)$ & $2.942 *(0.222-12.354)$ \\
\hline$\sigma_{u p}^{2}$ & $0.096 *(0.040-0.166)$ & $0.052 *(0.012-0.110)$ & $0.135 *(0.066-0.228)$ \\
\hline Observations & 4,606 & 4,606 & 4,606 \\
\hline Number of neighbourhoods & 124 & 124 & 124 \\
\hline DIC & 9342.01 & 9374.09 & 9384.296 \\
\hline
\end{tabular}

Notes: This table reports the results from Equations (1) through the the Markov Chain Monte Carlo (MCMC) Bayesian estimation. The pollution index of columns(1)-(3) is waste pollution, water pollution, and air pollution, respectively. The $95 \%$ credible interval(CI) for each coefficient is in parentheses and the symbol "*” represents statistical significance levels of $5 \%$ or better. 
Table 3. Estimation results on mover versus non-mover

(1) (2)

(3)

\section{Panel A: Mover sample}

1

2

4 Greenness*Pollution

5 Observations

7 Number of neighbourhoods

8 Panel B: Non-mover sample

9

10

11

12

13

14

15

$$
\begin{array}{cc}
0.309 *(0.048-0.572) & 0.092(-0.140-0.331) \\
-0.350 *(-0.480--0.220) & -0.300 *(-0.427--0.171) \\
1.178 *(0.765-1.539) & 0.924 *(0.681-1.168) \\
1,209 & 1,209 \\
116 & 116
\end{array}
$$$$
1.119 *(1.026-1.205)
$$$$
-0.307 *(-0.377--0.247)
$$$$
0.403 *(0.301-0.503)
$$$$
3,397
$$

123

$$
\begin{gathered}
1.192 *(1.075-1.304) \\
-0.316 *(-0.392--0.250) \\
1.172 *(1.019-1.317) \\
3,397 \\
123
\end{gathered}
$$

$0.470 *(0.042-0.871)$

$-0.234 *(-0.359--0.114)$

$1.014 *(0.614-1.415)$

1,209

116

$$
\begin{gathered}
1.197 *(1.087-1.310) \\
-0.213 *(-0.268--0.158) \\
0.439 *(0.359-0.521) \\
3,397 \\
123
\end{gathered}
$$

Table 4. Estimation results on heterogeneity and robustness test by social-spatial dimensions

\begin{tabular}{lccc} 
& $(1)$ & $(2)$ & $(3)$ \\
1.High school and below & $-0.475 *(-0.847--0.111)$ & $0.442 *(0.244-0.684)$ & $-0.579 *(-0.949--0.267)$ \\
2.College and above & $1.254 *(1.124-1.384)$ & $1.382 *(1.197-1.555)$ & $1.509 *(1.342-1.648)$ \\
3.Age above 40 & $-0.575 *(-0.665--0.490)$ & $-0.941 *(-1.256--0.636)$ & $-0.585 *(-0.717--0.451)$ \\
4.Age below 40 & $1.215 *(1.097-1.324)$ & $1.611 *(1.313-1.922)$ & $0.979 *(0.863-1.093)$ \\
$\begin{array}{l}\text { 5.Non-full time worker } \\
\text { 6.Full time worker }\end{array}$ & $0.451(-0.131-1.096)$ & $-0.413(-1.772-0.705)$ & $0.428 *(0.208-0.628)$ \\
7.Below the median of population & $1.043 *(0.909-1.186)$ & $1.357 *(1.211-1.493)$ & $0.541 *(0.403-0.662)$ \\
density & & & \\
8.Above the median of population & $1.156 *(0.918-1.381)$ & $0.929 *(0.814-1.033)$ & $-0.655 *(-0.787--0.506)$ \\
density & & & \\
9.Below the median of proportion & $0.058(-0.111-0.264)$ & $-0.113(-0.265-0.079)$ & $0.340 *(0.124-0.525)$ \\
of historical buildings & & & \\
10.Above the median of proportion & $0.600 *(0.403-0.770)$ & $0.479 *(0.296-0.656)$ & $0.129(-0.120-0.381)$ \\
of historical buildings & & & \\
11.Ordered logit model & $0.955 *(0.814-1.092)$ & $1.379 *(1.293-1.459)$ & $0.978 *(0.868-1.086)$ \\
12.Resident buffer = 500m & $0.745(-0.197-1.687)$ & $0.999 *(0.095-1.903)$ & $0.627(-0.601-1.854)$ \\
\hline
\end{tabular}

Notes: This table follows the baseline specifications but uses some sub-samples or other measurements to check the heterogeneity(rows 1-10) and robustness(rows 11-12) of our main results. The pollution index of columns(1)-(3) is waste pollution, water pollution, and air pollution, respectively. All regressions include the full set of covariates. The $95 \%$ credible interval(CI) for each coefficient is in parentheses and the symbol "*” represents statistical significance levels of $5 \%$ or better. 